\title{
Effects of Open Kinetic Chain Exercise for the Gastrocnemius and Tibialis Anterior Muscles on Balance
}

\author{
Song Yeon Yi, Young Ju Kim, Dong Yeop Lee, Jae Ho Yu, Jin Seop Kim, Soung Gil Kim, Ji heon Hong \\ Department of Physical therapy, College of Health Science, Sun Moon University, Republic of Korea
}

Purpose: This study investigated the effects of open kinetic chain (OKC) exercise for the gastrocnemius (GCM) and tibialis anterior (TA) muscles on static and dynamic balance and muscle strength.

Methods: We recruited 21 healthy participants, dividing them into 3 groups (GCM, TA, and non-exercise). Each group contains 7 participants. Two exercise groups (GCM and TA) performed OKC exercise with elastic bands twice per week for 4 weeks, while non-exercise group did nothing. We obtained the data for static and dynamic balance and muscle strength before and after the intervention. We used the Kruskal-Wallis test to compare and analyze the pre-post-intervention differences among the groups.

Results: For static balance, the stability index of the TA group was the lowest for the dynamic platform $(p<0.05)$. The dynamic balance of the TA group increased for the anterior and posteromedial directions $(p<0.05)$. The peak torque increased in the TA group for dorsiflexion (D/F) movement and in the GCM group for plantar flexion movement compared with the other groups, except for the left direction during $\mathrm{D} / \mathrm{F}(\mathrm{p}<0.05)$.

Conclusion: OKC exercises with elastic bands were effective for selectively increasing muscle strength. It is clinically thought that strength training for TA muscles will be effective among the muscles of the ankle.

Keywords: Dynamic balance, Static balance, Tibialis anterior muscle, Gastrocnemius muscle, Open kinetic chain, Muscle strengthening

\section{INTRODUCTION}

Balance, the ability to maintain the body's posture against gravity, is divided into static and dynamic. ${ }^{1}$ Static balance is the ability to maintain the center of gravity on a fixed base without swaying. ${ }^{2}$ Dynamic balance is the ability to maintain the center of gravity on a base when the body is in motion, exposed to external stimuli, or moving voluntarily. Balance improvement is an important goal in sports and exercise, and better balance can actively improve performance. ${ }^{3}$ Balance strategy is divided into ankle, hip, and stepping. Ankle muscle-strengthening exercises are applied to enhance balance and involve the muscles around the ankle joints, such as the tibialis anterior (TA), gastrocnemius (GCM), soleus, tibialis posterior, peroneus longus, and peroneus brevis. Plantar flexion (P/F), dorsiflexion (D/ F), inversion, and eversion for strengthening around the ankle are applied as isometric or resistance exercises. ${ }^{4}$ Static balance is the ability to maintain the center of gravity on stable platform while dynamic balance is the

Received Nov 16, 2021 Revised Dec 20, 2021

Accepted Dec 21, 2021

Corresponding author Ji heon Hong

E-mail hgh1020@hanmail.net ability to maintain the center of gravity on unstable platform on standing position. And previous study represent that these static and dynamic balance are directly related with standing balance. ${ }^{5}$

Standing balance is maintained by using an ankle or hip strategy, or both. ${ }^{6}$ Ankle strategy is the most often used for posture control and first appears in cases of slight perturbation. ${ }^{7}$ This strategy involves the primary recovery of standing balance through contraction of the ankle muscles. ${ }^{8}$ The alternative shaking in the anterior or posterior direction depends on the alternative activation of the TA and GCM muscles. The GCM begins to activate before the body falls in the forward direction, and the TA begins to activate before the body returns to the backward direction. ${ }^{9}$ Open kinetic chain $(\mathrm{OKC})$ exercise is defined as a movement that occurs in the body when free movement is available in the distal part. ${ }^{10}$ In this exercise, the distal areas move freely, while the proximal areas are fixed. This type of exercise plays an important role in strengthening the muscles of patients with limited range of motion. ${ }^{11}$ The OKC exercise is applied as a concen-

Copylight (C2021 The Korean Society of Physical Therapy

This is an Open Access article distribute under the terms of the Creative Commons Attribution Non-commercial License (https:// creativecommons.org/license/by-nc/4.o.) which permits unrestricted non-commercial use, distribution, and reproduction in any medium, provided the original work is properly cited. 
tric contraction that leads to higher traction and rotational forces. ${ }^{12}$ The effects of $\mathrm{OKC}$ exercise include an increase in concentric acceleration and excitability mechanoreceptors and the promotion of functional activities. ${ }^{13}$ According to a study examining the influence and effectiveness of $\mathrm{OKC}$ and closed kinetic chain (CKC) exercises on static balance control ability and muscle activity, the OKC exercise was more effective on the vastus medialis for 2-4 weeks of a muscle strength improvement intervention, and CKC exercise was more effective for muscle strength improvement with at least 4 weeks of exercise. ${ }^{10}$

Previous studies have reported the changes in static and dynamic balance related to muscle strength using $\mathrm{OKC}$ and CKC exercises. ${ }^{10-13} \mathrm{How}$ ever, studies on the effects of OKC exercise using select muscles on static and dynamic balance related to muscle strength are few. ${ }^{14}$ Therefore, in this study, we investigated muscle strength and static and dynamic balance after applying OKC exercises to the TA or GCM muscles in healthy adults, demonstrating the effects of the selective strength training of these muscles related to ankle strategy. To be more specific, focusing only on hip strategy muscles such as quadriceps and hamstring is limitation on previous studies. As known widely, hip strategy is usually generated after ankle strategy. To overcome the limitation of previous study, we focused on exercising and strengthening TA and calf muscle which are mainly used for ankle strategy to suggest clinical data.

\section{METHODS}

\section{Subjects}

We conducted this study on 21 healthy adult men and women from S University in Asan, South Korea. Each participant received a thorough explanation of the purpose and methods of this study and a preliminary test. After giving their written informed consent, the participants received an explanation of the experiment's purpose. We excluded those who had a musculoskeletal disease in the lower extremity (LE), a history of injury or neurological disease, and balance problems. Each group (GCM, TA, Nonexercise) contains 7 participants. Both GCM and TA group performed exercise as intervention, while non-exercise group did nothing. P/F exercise is conducted for GCM group, D/F is conducted for TA group by using TheraBand before and after measurement while non-exercise group participants only performed measurements. This study, which compared the GCM and TA groups in a pre- and post-test randomized control group design, was approved by the Institutional Review Board of Sunmoon University (SM-202005-027).
Table 1. General participant characteristics

$(n=21[$ male $=9$, Female $=12])$

\begin{tabular}{lccc}
\hline Division & $\begin{array}{c}\text { GCM Group } \\
(\mathrm{n}=7)\end{array}$ & $\begin{array}{c}\text { TA Group } \\
(\mathrm{n}=7)\end{array}$ & $\begin{array}{c}\text { Non-Exercise } \\
\text { Group }(\mathrm{n}=7)\end{array}$ \\
\hline Age $(\mathrm{yr})$ & $22.1 \pm 0.7$ & $32.9 \pm 0.7$ & $23.3 \pm 0.8$ \\
Height $(\mathrm{cm})$ & $161.5 \pm 5.3$ & $162.9 \pm 7.7$ & $174 \pm 4.1$ \\
Weight $(\mathrm{kg})$ & $56.1 \pm 6.2$ & $56.7 \pm 10.6$ & $71.5 \pm 9.8$ \\
\hline
\end{tabular}

Mean \pm SD

GCM: gastrocnemius, TA: tibialis anterior, SD: standard deviation.

\section{Procedure}

Participants were involved in the experiment for a total of 4 weeks. To prevent injuries during exercise and examination, participants performed 3 min of warm-up exercises and 10 minutes of stretching before the exercise and examination. The groups were divided into GCM exercise, TA exercise, and non-exercise. Before starting the experiment, we measured the participants' height and weight using an autonomic body mass indexmeasuring radiometer (BSM 370, INBODY, Korea). Participants' general characteristics are shown in Table 1. We measured the dynamic and static balance and muscle strength twice before and after the intervention. An elastic band was used for the exercises, and participants performed 3 sets (10 reps and a 30-seconds break) twice a week for 4 weeks. GCM group performed maximal plantar flexion $\left(45-50^{\circ}\right)$ and TA group performed maximal dorsiflexion $\left(15-20^{\circ}\right)$ intervention 3 sets a day, two times a week, for 4 weeks. Only one therapist manages whole interventions by using different thera-bands which has various intensity. Intensity of intervention is enhanced grad-ually every week (Figure 1).

\section{Measurement}

We used elastic bands (Hygienic Corp, Akron, OH, USA) for the OKC exercises. An elastic band is rarely influenced by gravity because muscle strength is exerted by resisting against the band's contracting force. ${ }^{16}$ The participants performed the exercises for 4 weeks. In this program, we adjusted the intensity of the bands every week. We set the tension to $170 \%$ to obtain the most significant exercise effects. ${ }^{17}$ The participants performed the exercise program by doing 3 sets of 10 reps, taking a 30 seconds break after each set. In the GCM exercise, the participants sat on a mat and erected their waist in $90^{\circ}$ with their legs straight. After aligning the ankle angle to $90^{\circ}$, the participants grabbed and placed the elastic bands in 2/1 above from the center of the sole and performed $\mathrm{P} / \mathrm{F}$ to $40^{\circ}$. In the TA exercise, the participants sat on a mat, erected their waist in $90^{\circ}$ with their legs straight, and aligned their ankle angle to $90^{\circ}$. The participants grabbed and placed elastic bands at the center of the top of the feet and performed 


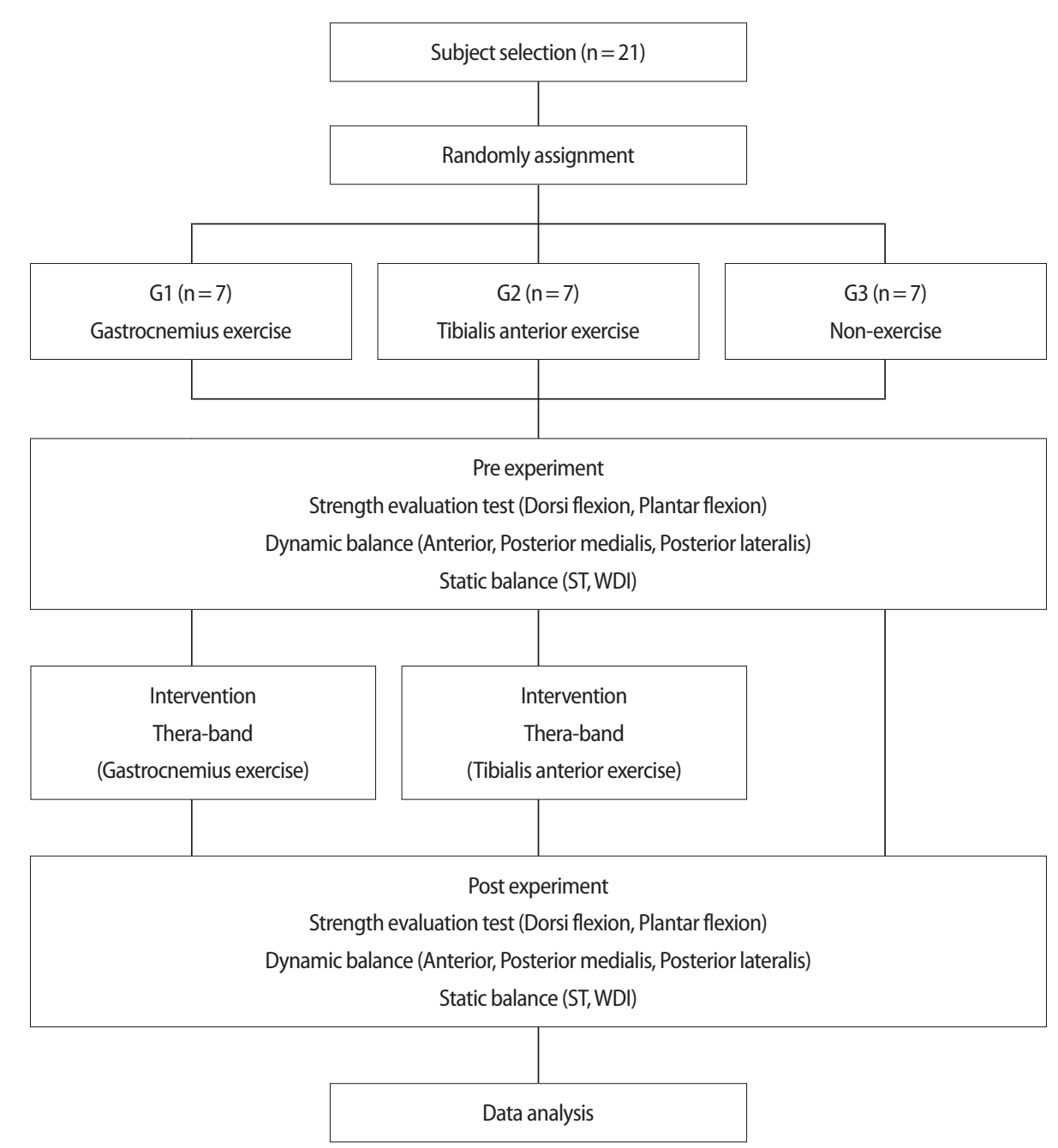

Figure 1. Research process flow chart.

$\mathrm{D} / \mathrm{F}$ to $20^{\circ}$. Once a week, the participants used 3 types of bands with different tensions, depending on the exercise intensity. The participants used green, blue, and black bands; the black bands had the strongest tension, followed by the blue and green. ${ }^{17}$

We measured the static balance (stability index $[\mathrm{ST}]$ and weight distribution index [WDI]) using the Tetrax ${ }^{\circledR}$ tetra-ataxiometric posturography system (Sunlight Medical Ltd., Ramat Gan, Israel). We used 4 postures in the study, evaluating static balance by measuring during the following conditions: eyes open in the stable platform (NO), eyes closed in the stable platform (NC), eyes open in the dynamic platform (PO), and eyes closed in the dynamic platform (PC) (Figure 2). ST showed stability by measuring the changes in posture depending on the changes in weight, and WDI indicated the weight bearing as a percentage. For the measurement posture, the participants opened or closed their eyes in the stable and unstable platforms. In both platforms, the participants were told to maintain a pos- ture for 32 seconds and stare at a dot on the wall $1 \mathrm{~m}$ away. Participants with lower ST and WDI data had higher balance.

We measured the dynamic balance using the FMS Y-Balance Test (YBT) Kit (PerformBetter West Warwick, RI, USA). The participants stood on one leg at the center of the YBT and pushed the block to the anterior (ANT), posteromedial (PM), or posterolateral (PL) directions with the other leg (Figure 3). Because shoes can influence stability during the measurements, the participants performed in their bare feet. We measured the participants 3 times in each direction after they had twice practiced each. We measured the total reach distance by reading a tape measure from the farthest reach of the foot. We measured the participants again without recording the attempt if they failed to maintain the posture in the platform.

To evaluate muscle strength, we used the Cybex dynamometer (CSMI Solutions, Stoughton, MA, USA). In all tests, we asked the participants to lie down straight and bend the thigh far from the thigh stabilizer, hip, and 

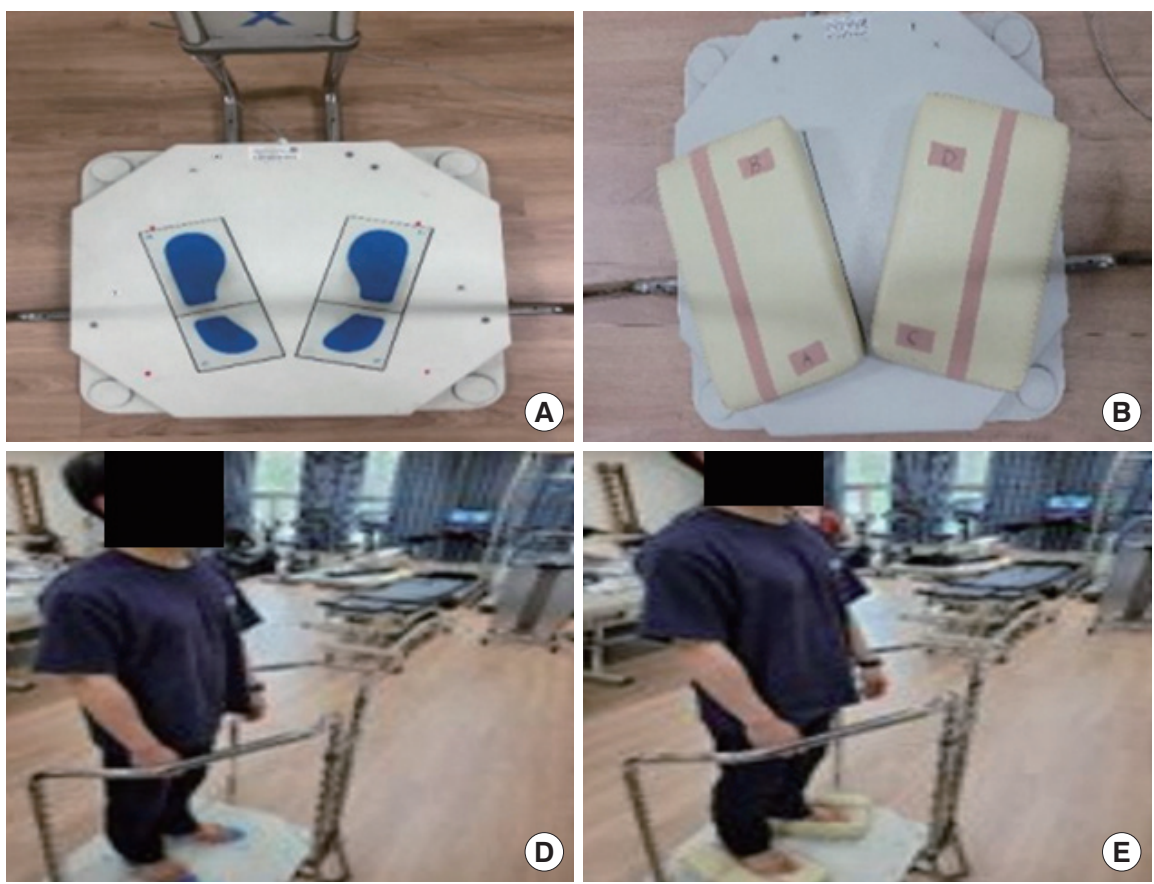
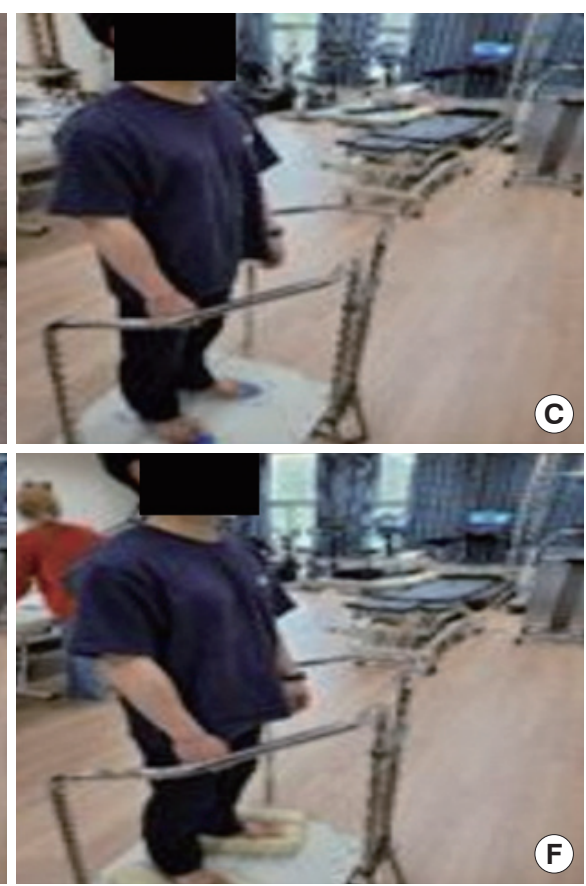

Figure 2. Tetrax. (A) force plate, (B) Tetrax pillow, (C) normal eyes open, (D) normal eyes closed, (E) pillow with eyes open, (F) Pillow with eyes closed.
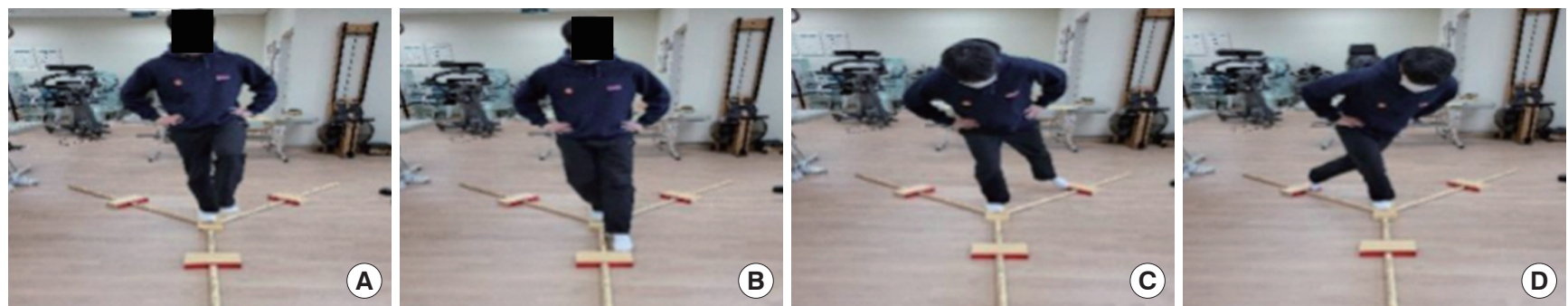

Figure 3. Y-Balance Test. (A) starting, (B) anterior, (C) posteromedial, (D) posterolateral.

knee in $90^{\circ}$. The related legs and arms were fixed to the end of the thigh and pelvis. The participants conducted up to 3 practice tests. After practicing in submaximal effort, the participants performed $\mathrm{P} / \mathrm{F}$ and $\mathrm{D} / \mathrm{F}$ twice in each direction. The participants performed 2 sets 4 times in $100 \%$ seconds for 20 seconds, then took a break for 15 seconds. We used the average peak torque for 3 attempts in the statistical analysis. ${ }^{18}$

\section{Statistical analysis}

All statistical analysis used SPSS statistical software (version 20.0, IBM Corp. Armonk, NY, USA). For the static and dynamic balance and muscle strength data, we used the difference between the postintervention and pre-intervention values. The normality test did not meet the between-group normality for the Shapiro-wilks test. For this reason, ST, WDI, YBT, and the change of maximum torque between groups were compared using the Kruskal-Wallis test, which is a non-parametric statistical method. Bonferroni correction method post hoc tests were used to assess data differences between groups.

\section{RESULTS}

\section{Static balance}

The pre-post variation of the ST, PO, and PC for each group showed a significant difference, whereas the ST of NO and NC and the WDI showed no significant differences (Table 2). The ST of the GCM and TA groups with PO improved compared with the non-exercise group. The ST of the TA group with PC was the lowest compared with the other groups.

\section{Dynamic balance}

In the pre-post variation in all groups, the participants showed significant differences in reach distance for all directions $(\mathrm{p}<0.05)$ (Table 3$)$. The in- 
crease in movement distance for the ANT and PM directions in the TA group was the largest compared with the other groups. The increase in

Table 2. Static balance, by intervention

$(n=21)$

\begin{tabular}{|c|c|c|c|c|}
\hline & Position & Group & Mean \pm SD & $\mathrm{p}$ \\
\hline \multirow[t]{12}{*}{ ST } & \multirow[t]{3}{*}{ NO } & GCM & $0.61 \pm 1.75$ & \multirow[t]{3}{*}{0.746} \\
\hline & & TA & $-0.60 \pm 3.79$ & \\
\hline & & Non-exercise & $0.72 \pm 4.61$ & \\
\hline & \multirow[t]{3}{*}{ NC } & GCM & $3.39 \pm 4.12$ & \multirow[t]{3}{*}{0.089} \\
\hline & & TA & $1.24 \pm 4.86$ & \\
\hline & & Non-exercise & $6.47 \pm 8.57$ & \\
\hline & \multirow[t]{3}{*}{$\mathrm{PO}$} & GCM & $-5.29 \pm 4.14$ & \multirow[t]{3}{*}{$0.017^{\star}$} \\
\hline & & TA & $-6.77 \pm 3.05$ & \\
\hline & & Non-exercise & $1.20 \pm 5.11$ & \\
\hline & \multirow[t]{3}{*}{ PC } & GCM & $-4.21 \pm 2.33$ & \multirow[t]{3}{*}{$0.003^{*}$} \\
\hline & & TA & $-4.31 \pm 2.77$ & \\
\hline & & Non-exercise & $1.72 \pm 1.72$ & \\
\hline \multirow[t]{12}{*}{ WDI } & \multirow[t]{3}{*}{ NO } & GCM & $0.69 \pm 2.08$ & \multirow[t]{3}{*}{0.546} \\
\hline & & TA & $1.10 \pm 3.83$ & \\
\hline & & Non-exercise & $-4.09 \pm 3.76$ & \\
\hline & \multirow[t]{3}{*}{ NC } & GCM & $6.47 \pm 3.73$ & \multirow[t]{3}{*}{0.228} \\
\hline & & TA & $4.21 \pm 4.12$ & \\
\hline & & Non-exercise & $-3.31 \pm 7.52$ & \\
\hline & \multirow[t]{3}{*}{$\mathrm{PO}$} & GCM & $-1.02 \pm 1.51$ & \multirow[t]{3}{*}{0.597} \\
\hline & & TA & $-1.42 \pm 1.86$ & \\
\hline & & Non-exercise & $0.72 \pm 4.61$ & \\
\hline & \multirow[t]{3}{*}{ PC } & GCM & $0.87 \pm 0.97$ & \multirow[t]{3}{*}{0.163} \\
\hline & & TA & $0.75 \pm 1.54$ & \\
\hline & & Non-exercise & $0.59 \pm 1.30$ & \\
\hline
\end{tabular}

Mean \pm SD.

SD: standard deviation, ST: stability index, WDI: weight distribution index, NO: normal eyes open, NC: normal eyes closed, PO: pillow with eyes open, PC: pillow with eyes closed, GCM: gatrocnemius, TA: tibialis anterior. ${ }^{*} \mathrm{p}<0.05$. distance for the PL direction was greatest in the GCM group (Figure 4).

\section{Muscle strength}

The peak torque for the isokinetic muscle strength variation for each group is shown in Table 4. There was a significant difference among the groups for ankle movements. For the P/F movement, the increase in peak torque was the largest in the GCM group $(\mathrm{p}<0.05)$. For the D/F movement, the peak torque in the TA group increased the most $(\mathrm{p}<0.05)$ (Figure 5).

\section{DISCUSSION}

This study investigated the effects of muscle strength improvement with OKC exercise with the TA and GCM muscles for static-balancing and dynamic-balancing ability. The peak torque was increased in the TA group Table 3. Dynamic balance, by intervention $(n=21)$

\begin{tabular}{lllc}
\hline & Group & Mean \pm SD & $p$ \\
\hline ANT & GCM & $0.85 \pm 0.63$ & $0.002^{*}$ \\
& TA & $1.37 \pm 0.92$ & \\
& Non-exercise & $-0.62 \pm 0.94$ & \\
PM & GCM & $0.14 \pm 0.12$ & $0.035^{*}$ \\
& TA & $0.34 \pm 0.32$ & \\
PL & Non-exercise & $-0.07 \pm 0.21$ & \\
& GCM & $0.37 \pm 0.12$ & $0.005^{*}$ \\
& TA & $0.15 \pm 0.37$ & \\
& Non-exercise & $-0.01 \pm 0.03$ & \\
\hline
\end{tabular}

mean \pm SD.

ANT: anterior direction, PM: posteromedial direction, PL: posterolateral direction, GCM: gastrocnemius, TA: tibialis anterior. ${ }^{*} \mathrm{p}<0.05$.

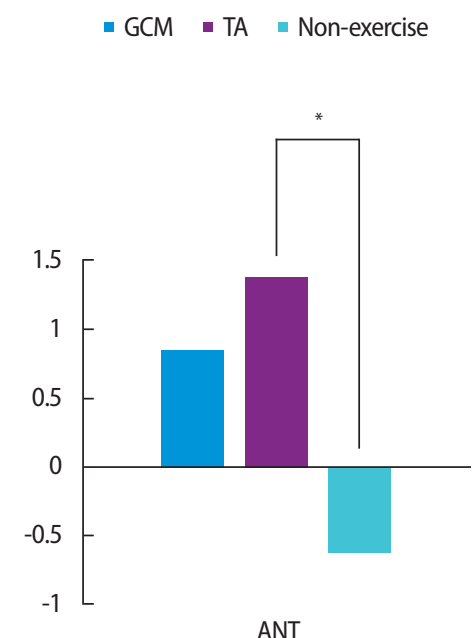

ANT

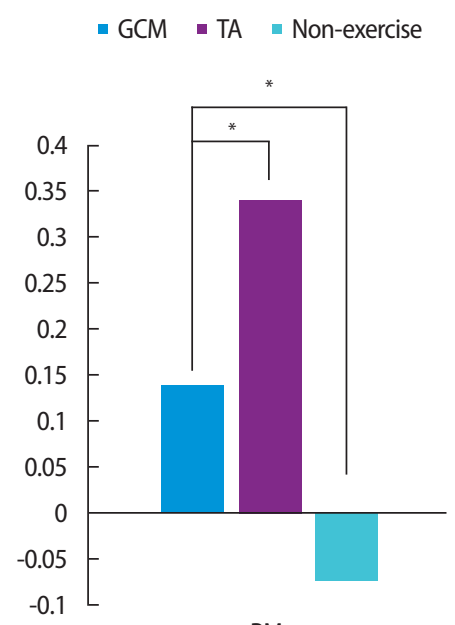

PM

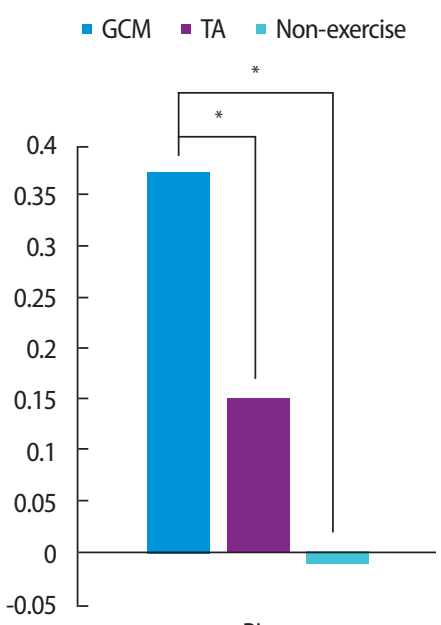

PL

Figure 4. Comparison of dynamic balance. 


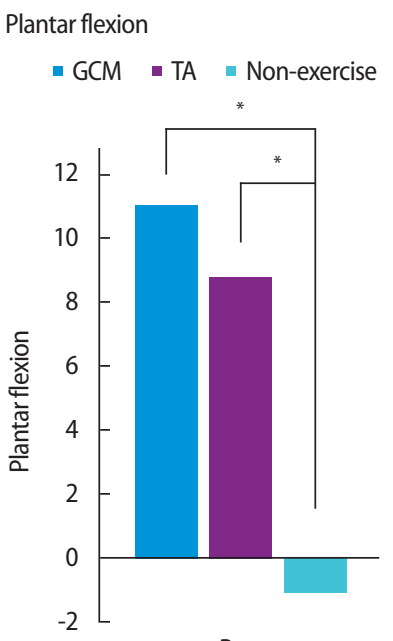

Rt

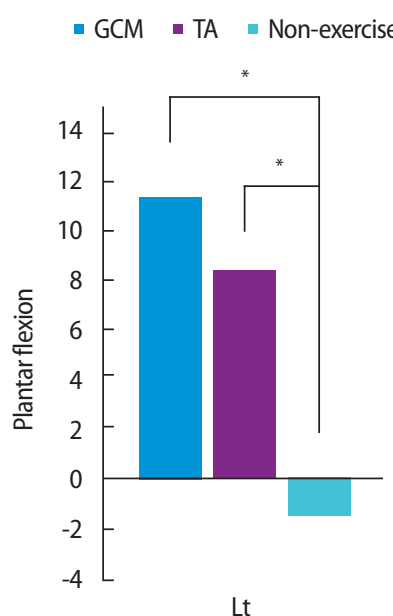

Lt

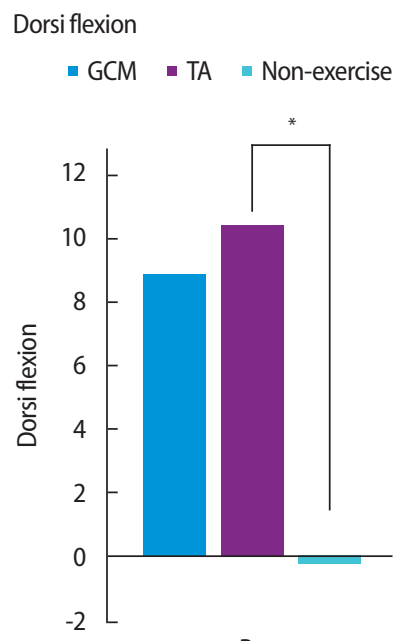

Rt

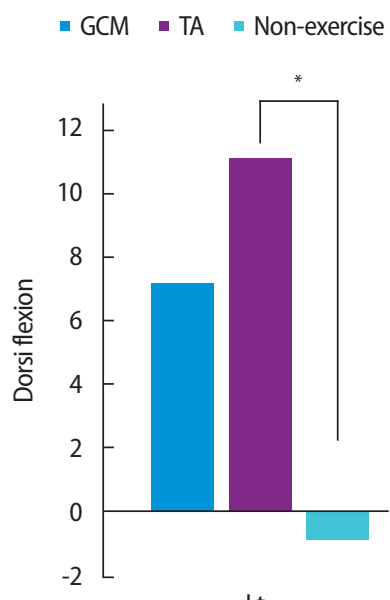

Lt

Figure 5. Comparison of muscle strength.

\begin{tabular}{|c|c|c|c|c|}
\hline & Position & Group & Mean $\pm S D$ & $\mathrm{p}$ \\
\hline \multirow[t]{6}{*}{$\mathrm{P} / \mathrm{F}$} & Right & GCM & $11.04 \pm 7.04$ & $0.006^{*}$ \\
\hline & & TA & $8.85 \pm 9.88$ & \\
\hline & & Non-exercise & -1.12 & \\
\hline & Left & GCM & $11.42 \pm 8.32$ & $0.036^{*}$ \\
\hline & & TA & $8.42 \pm 11.12$ & \\
\hline & & Non-exercise & $-1.42 \pm 3.40$ & \\
\hline \multirow[t]{6}{*}{$\mathrm{D} / \mathrm{F}$} & Right & GCM & $7.14 \pm 3.48$ & 0.001 * \\
\hline & & TA & $10.43 \pm 5.16$ & \\
\hline & & Non-exercise & $-0.14 \pm 2.47$ & \\
\hline & Left & GCM & $11.14 \pm 5.04$ & $0.003^{*}$ \\
\hline & & TA & $7.28 \pm 5.12$ & \\
\hline & & Non-exercise & $-0.82 \pm 1.34$ & \\
\hline
\end{tabular}

Mean \pm SD.

P/F: plantar flexion, D/F: dorsiflexion, GCM: gastrocnemius, TA: tibialis anterior. ${ }^{*} p<0.05$.

for $\mathrm{D} / \mathrm{F}$ and in the GCM group for $\mathrm{P} / \mathrm{F}$. The most important result in this study was for static balance. No differences existed in the WDI after muscle strengthening, but the ST decreased the most in the TA group. The dynamic balance of the TA group improved for the ANT and PM directions and the PM direction improved for the GCM group. Therefore, we suggest that selective strengthening using OKC exercises for the ankle muscles can improve balance. The effect on the TA muscle is stronger than that for the GCM muscle.

OKC exercise can play an important role in strengthening muscles. ${ }^{11,18}$ Elastic bands can have a significant effect on improving ankle strength. ${ }^{20}$ In a previous study on the effects of $\mathrm{OKC}$ and $\mathrm{CKC}$ exercises on muscle strength, the strength of the TA muscle increased with the OKC exercises during the first 2 weeks of the intervention. ${ }^{19}$ In contrast, the GCM strength

increased with both the $\mathrm{OKC}$ and $\mathrm{CKC}$ exercises after 4 weeks, but $\mathrm{CKC}$ exercise was more effective. Therefore, the OKC exercise can have a faster muscle-strengthening effect. These results represent that OKC exercise using TheraBand on TA and GCM is more efficient than CKC exercise for both static and dynamic balance within 4 weeks. Which means OKC exercise using TheraBand is more effective to increase short term balance.

Muscle strength is gradually reduced because of the long-term fixation or bed life due to muscle tissue, nerve, and joint lesions, as well as to the increase in age; this strength is essential for improving the functions of walking and daily movement. ${ }^{21}$ Balance exercises are done to enhance the LE, in-cluding the ankle. Training for proprioception, including strengthening the ankle muscles, has a positive influence on the static balance of healthy individuals. ${ }^{24}$ A previous study has compared the activation of the TA and the lateral and medial GCM muscles under different static balance conditions for healthy adults, finding that the GCM muscle was activated mainly for balancing in a resting position, while the activation of the TA and GCM muscles increased during various stimulations of proprioception. ${ }^{23}$ In our study, we found a significant difference when using a pillow during static balance (the pillow caused dynamic confusion about balance). Therefore, the TA group had a higher improvement in balance compared with the other groups.

Static and dynamic balance exercises are generally performed on unstable ground ${ }^{26}$ because standing on an unstable surface stimulates the use of proprioceptive signals coming from around the ankle and, in this way, helps with ankle stability. In other words, it is difficult to maintain balance on unstable ground. Therefore, in this study, we found a significant difference when a pillow was used during static balance. Afferent stimuli related to balance involve the visual, vestibular, and somatosensory 
systems. Vision plays a key role in locating the position of the body in the surrounding environment, in stabilizing the posture, and in the expected response. ${ }^{22}$ The length and speed of swaying in the static balance for the ankle and LE increase when there is no visual information. ${ }^{23}$ When sight is blocked, the body uses other proprioceptive senses. The proprioceptive sense of the ankle enables the integration with other sensory information for controlling posture and balance, thus playing an essential role in balance. ${ }^{25}$ Healthy adults can maintain their balance by depending on other senses when their vision is blocked.

A previous study has reported that YBT is influenced by different anatomical factors for each direction. ${ }^{27}$ In the posterior direction, hip joint flexion plays an important role; performance decreased in the patients with low back pain for the posterior direction, while it did not decrease in the ANT direction, implying that the rear direction has a significant correlation with the core. The anterior shin muscle worked when the body was agitated backward, and the iliac muscle was activated as the center of the body moved forward. ${ }^{28}$ Another study has reported that, to maintain balance, the GCM muscle was activated for the ANT swing, and the TA muscle was activated for the posterior swing. ${ }^{29}$ In addition, the strengthening of the $\mathrm{P} / \mathrm{F}$ and invertor muscle is correlated with improvement of the ANT direction. Previous research has reported the correlation of the ANT direction with ankle stability.

The current study has several limitations. First, the participants were limited to healthy men and women in their 20 seconds. Therefore, the results cannot be generalized to other age groups. Second, the effects of a long-term intervention could not be determined due to our short intervention time. Thus, it is necessary to study other populations and apply additional intervention methods for comparison in future research. In conclusion, the OKC exercise during a 4-week intervention enhanced muscle strength and balance. Selective strengthening of the TA muscles was most effective for increasing balance.

In conclusion, our experiment represents two significant factors. First, OKC exercise using TheraBand is more effective than Calisthenic OKC exercise. Second, exercise affect TA more than GCM. We can infer that selecting right exercise on right selective structure depends on subject's capacity of exercise might be adapted for therapeutic exercise and these data will served as baseline for on coming clinical research.

\section{ACKNOWLEDGEMENTS}

This work was supported by the National Research Foundation of Korea
(NRF) grant funded by the Korea government(MSIT) (2020R1C1C1012483).

\section{REFERENCES}

1. Kim HS. The exercise of the human body. 3rd ed. Seoul, Hyunmoonsa, 1996:246-86

2. Berger L, Klein C, Commandeur M. Evaluation of the immediate and midterm effects of mobilization in hot spa water on static and dynamic balance in elderly subjects. Ann Readapt Med Phys. 2008;51(2):90-5.

3. Han J, Anson J, Waddington G et al. The role of ankle proprioception for balance control in relation to sports performance and injury. BioMed Res Int. 2015;2015:842804.

4. Shumway-Cook A, Horak FB. Assessing the influence of sensory interaction on balance: suggestion from the field. Phys Ther. 1986;66(10): 1548-50.

5. Kim JH. A study on the correlation between static, dynamic standing balance symmetry and walking function in stroke. J Kor Phys Ther. 2012; 24(2):73-81.

6. Horak FB, Nashner LM. Central programming of postural movements: adaptation to altered supportsurface configurations. J Neurophysiol. 1986;55(6):1369-81.

7. Shumway-Cook A, Woollacott MH. Motor control: theory and practical application, Philadelphia, Lip-pincott Williams and Wilkens, 1995: 188-90.

8. Horak FB, Shupert CL, Mirka A. Components of postural dyscontrol in the elderly: a review. Neurobiol Aging. 1989;10(6):727-38.

9. Almeida GL, Carvalho RL, Talis VL. Postural strategy to keep balance on the seesaw. Gait Posture. 2006;23(1):17-21.

10. Kwon YJ, Park SJ, Kim K. The effect of open and closed chain exercise on lower extremity muscle activity in adults. J Korean Soc Phys Med. 2012;7 (2):173-82.

11. Jang JW. Changes in muscular activity of the quadriceps in the open and closed mechanical movement. Korea University. Dissertation of Master's Degree. 2003.

12. Kim Y, Kim T, Oh D. The effects of closed kinetic chain exercise and open kinetic chain exercise in improving the balance of patients with hemiplegia. J Korean Acad Orthop Man Physi Ther. 2009;15(1):22-31.

13. Prentice WE. Techniques in musculoskeletal rehabilitation, Paju, Koonja, CHAPTER 12. Open-versus closed kinetic chain exercises in rehabilitation, 2005:203.

14. Kwon YJ. The effect of dynamic balance recovery and muscle activation by exercises on normal adult. Daegu University. Dissertation of Master's Degree. 2009.

15. López-Plaza D, Juan-Recio C, Barbado D et al. Reliability of the star excursion balance test and two new similar protocols to measure trunk postural control. PM\&R. 2018;10(12):1344-52.

16. Lee D. Effects of theraband exercise programs on strength, balance and proprioception in elderly. J Korean Soc Integr Med. 2014;2(4):1-8.

17. Smith BI, Docherty CL, Simon J et al. Ankle strength and force sense after a progressive, 6-week strength-training program in people with functional ankle instability. J Athl Train. 2012;47(3):282-8.

18. Kim YJ. Effects of open and closed chain exercise on dynamic balance and muscle activity changes in normal adults. Daegu University. Disser- 
tation of Master's Degree.

19. Kwon YJ, Park SJ, Jefferson J et al. The effect of open and closed kinetic chain exercises on dynamic balance ability of normal healthy adults. J Phys Ther Sci. 2013;25(6):671-4.

20. Jeong M. The effects of the thera-band exercise program on lower extremities muscle strength and balance in rural female farmers. J Korean Acad Rural Health Nurs. 2006;1(1):49-58.

21. Yang HS, Lee KW. Comparison of the balance relations between healthy subjects and patients with chronic low back pain. Phys Ther Korea. 2002;9(2):1-17.

22. Macedo C, Gazzola JM, Ricci NA et al. Influence of sensory information on static balance in older patients with vestibular disorder. Braz J Otorhinolaryngol. 2015;81:50-7.

23. Kim S, Kim W. Effect of ankle range of motion (ROM) and lower-extremity muscle strength on static balance control ability in young adults: a regression analysis. Med Sci Monitor. 2018;24:3168.

24. Karakaya MG, Rutbil H, Akpinar E et al. Effect of ankle proprioceptive training on static body balance. J Phys Ther Sci. 2015;27(10):3299-302.

25. Woo YG, Park JW, Choi JD et al. Characteristics of lower leg muscle activity under various conditions during static balance control in normal adults. Phys Ther Korea. 2004;11(2):35-45.

26. Kiers H, Brumagne S, Van Dieen J et al. Ankle proprioception is not targeted by exercises on an unstable surface. Eur J Appl Physiol. 2012;112 (4):1577-85.

27. Hooper TL, James CR, Brismée J et al. Dynamic balance as measured by the Y-balance test is reduced in individuals with low back pain: a crosssectional comparative study. Phys Ther Sport. 2016;22:29-34.

28. Woo Y, Park J, Choi J et al. Electromyographic activities of lower leg muscles during static balance control in normal adults. Phys Ther Korea. 2004;11(2):35-45.

29. Kim C, Jung H, Lee B et al. Characteristics of balance and muscle activation responded to dynamic motions in anterior-posterior and mediallateral directions. J Biomed Eng Res. 2013;34(4):212-7. 